\title{
Application of Dual-Frequency Ultrasound for Treating the Ulcerative Necrobiosis Lipoidica
}

\author{
Seok Won Hong ${ }^{1}$ \\ Eun Soo Park ${ }^{1}$ \\ Seok Hwan Kim ${ }^{1}$ \\ Min Jung Jung²
}

${ }^{1}$ Department of Plastic and Reconstructive Surgery, Soonchunhyang University College of Medicine, Bucheon Hospital, Bucheon, Korea

${ }^{2}$ Department of Pathology, Soonchunhyang University, College of Medicine, Bucheon, Korea
Received November 26, 2019

Accepted December 8, 2019

\section{Correspondence}

Eun Soo Park

Department of Plastic and Reconstructive Surgery, Soonchunhyang University Bucheon Hospital, 1174 Jomaru-ro, Wonmi-gu, Bucheon 14584, Korea

Tel.: +82-32-621-5319

Fax: $+82-32-621-5016$

E-mail: peunsoodschmc.ac.kr

(C) Korean Society for Laser Medicine and Surgery

(c) This is an open access article distributed under the terms of the Creative Commons Attribution NonCommercial License (http://creativecommons.org/ licenses/by-nc/4.0) which permits unrestricted noncommercial use, distribution, and reproduction in any medium, provided the original work is properly cited.
Necrobiosis lipoidica (NL) is a rare, idiopathic, chronic granulomatous inflammatory disease of collagen degeneration with the risk of ulceration. Many procedures have been proposed to treat this rare disease. In this study, we applied LDM ${ }^{\circledR}$-MED for the management of $\mathrm{NL}$, and this condition in our patient was chronic and refractory to other therapeutic options. To the best of our knowledge, no study has explored treatment of NL using ultrasound. Our results suggest that application of $\mathrm{LDM}^{\circledR}$-MED seems to be an effective treatment option for NL. Long-term and systematic studies are needed to determine whether such application of LDM ${ }^{\circledR}$-MED will be an innovative and effective treatment option for NL and various kinds of chronic wounds.

\section{Key words}

Necrobiosis lipoidica; Necrobiosis lipoidica/therapy; Ulceration; Ultrasound 


\section{INTRODUCTION}

Necrobiosis lipoidica (NL) is a rare, idiopathic, chronic granulomatous inflammatory disease. It is a kind of collagen degeneration disease with the risk of ulceration. In the 1960s to 1980s, studies that set the basis for understanding this disease process were conducted, revealing what we now know about this disease. ${ }^{1}$ However, clinical progression of NL is still difficult to predict. There are no clinical trials to guide the efficacy of treatment options. To offer choices of treatment options to patients, many subsequent analysis and case reports have been used to build the knowledge base about this disease..$^{1-3}$

Many procedures have been proposed to treat NL. However, no treatment has been proven to be effective. For some, topical intra-lesional and systemic corticosteroid remains the first-line treatment. Moreover, ultraviolet light therapy and calcineurin inhibitors have been suggested as choices of treatment for $\mathrm{NL}^{4-6}$

In this study, we present a case in which a young patient with NL who did not respond to conventional treatment was treated with the application of ultrasound. We applied dual-frequency ultrasound therapy in the management of $\mathrm{NL}$. For the treatment, we used dual-frequency $(3 \mathrm{MHz}$ and $10 \mathrm{MHz}$ frequencyl ultrasound device named $\mathrm{LDM}^{\circledR}$ MED. This device can lead to cellular activation through focal, dynamic micro-oscillation. It is especially effective for chronic wounds. ${ }^{7}$ To the best of our knowledge, studies using ultrasound therapy for treating NL have not been reported yet. Our results suggest that LDM ${ }^{\circledR}$-MED could be used as an effective treatment option for NL.

\section{CASE REPORT}

Our patient was a 20-year-old female who presented chronic and non-healing ulcerative skin lesion on her right lower leg. She did not know why and when the skin lesion developed. She had a family history of diabetes mellitus (DM). She was diagnosed with type 2 DM five years ago. A skin lesion occurred as a reddish papule initially. It slowly evolved into a chronic ulcer. She had been treated with topical intralesional and systemic corticosteroids at a local clinic for more than six months. After a few months of refractory treatment, there was no effect. It became chronic ulcerative wound. For evaluation, punch biopsy was done. It was diagnosed with necrobiosis lipoidica (Fig. 1). The patient was transferred to our clinic for treatment. She had been treated with dual-frequency
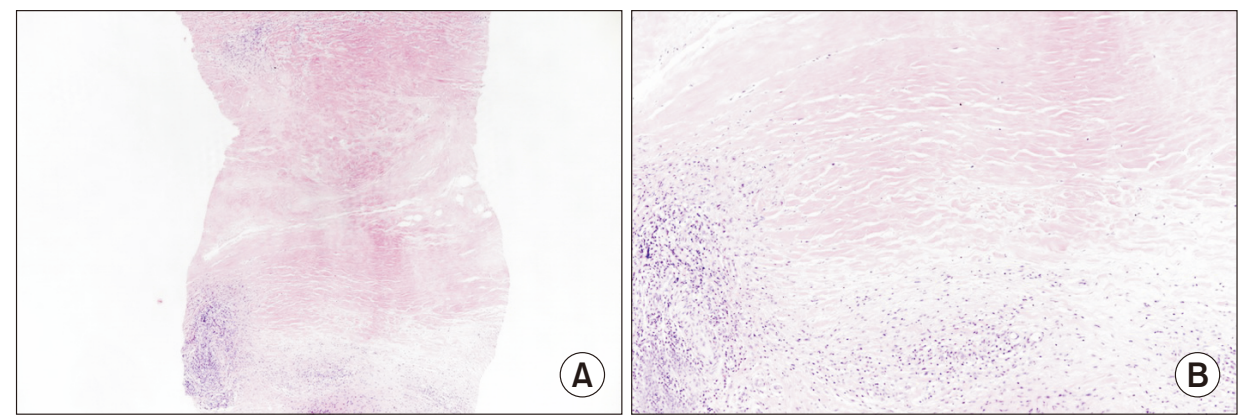

Fig. 1. Pathologic images of Necrobiosis lipoidica. (A) This low-power view of a punch biopsy shows a broad zones of necrobiotic collagen in the almost entire dermis. There is a perivascular inflammatory infiltrate at the edge (Hematoxylin and Eosin, $\times 40$ ). (B) The degenerated necrobiotic collagen is eosinophilic and the inflammatory infiltrate is composed of an admixture of lymphocytes and histiocytes (Hematoxylin and Eosin, $\times 100)$.
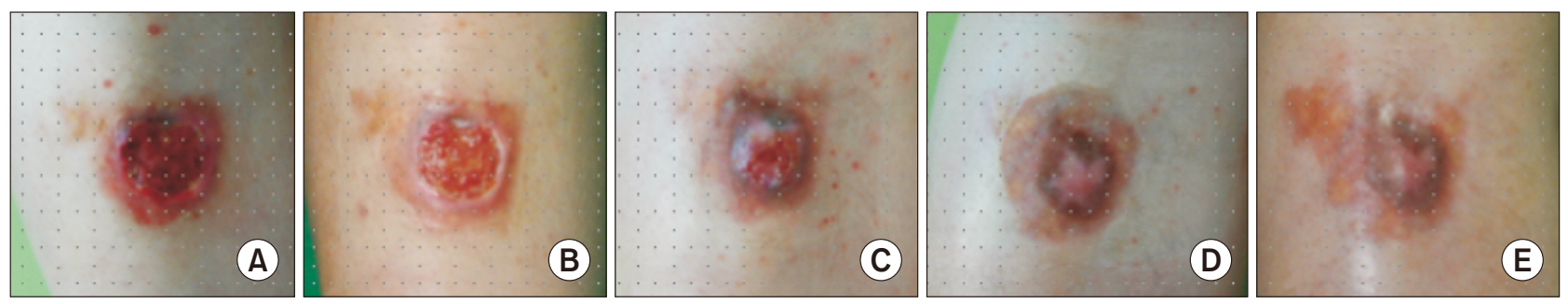

Fig. 2. Treatment progress images of ulcerative necrobiosis lipoidica. (A) Clinical photographs of the ulcerative NL on day 1 before LDM ${ }^{\circledR}-M E D$ treatment. (B) 4 days after 4 treatment sessions. (C) 3 weeks after 15 treatment sessions. (D) 7 weeks after 28 treatment sessions. (E) 3 months after total 38 treatment sessions of LDM $^{\circledR}$-MED. 


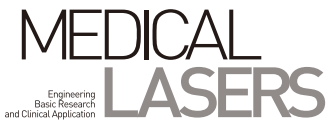

ultrasound (LDM ${ }^{\circledR}$-MED) according to the author's protocol. The wound was at the anterior aspect of the lower leg. It was a $2.3 \times 3.4 \mathrm{~cm}$ sized reddish ulceration without infection sign. Slight yellowish discharge was seen. She had taken thorough glycemic control by close consultation with the department of endocrinology. Prophylactic intravenous cefazolin 1,000 mg was administered twice a day for two weeks followed by every day for the next 3 weeks ( 5 days in a row per week) and every other day for the next 4 weeks ( 3 days per week). She had a total of 38 sessions for a 3-month period (Fig. 2). At each treatment session, the patient was evaluated by the author and clinical digital photographic documentation was carried out. In order to obtain a visual evaluation, we measured the ulcerative area. Treatment had been continued until the ulcerative lesion was fully re-epithelized. The author added 10 sessions (2 sessions per week) after re-epithelization of the wound to improve scar remodeling.

\section{DISCUSSION}

Necrobiosis lipoidica (NL) is a rare idiopathic granulomatous disease of collagen degeneration with risk of ulceration. This disease has typically been associated with diabetes. However, the strength of such association has been questioned over time. ${ }^{1,2}$ Besides, other systemic diseases such as thyroid disease and inflammatory disease are often associated with NL. In the 1960s to 1980s, studies that set the basis for understanding this disease process had been conducted. They have revealed what we now know about this disease. 'Although the incidence of $\mathrm{NL}$ in diabetes patient is only $0.3 \%$ to $1.2 \%,{ }^{2,3}$ clinical progression of $\mathrm{NL}$ is still difficult to predict as it varies with individuals. Effects of other systemic diseases on the clinical process of NL remain controversial. Many unknowns aspects require more studies. We need to find its causes and treatment options that are both efficacious and consistent. ${ }^{8}$

As one of unknown aspects that require more studies, the pathogenesis of NL has been proposed to be collagen degeneration for many years. However, the exact pathogenesis of NL remain unknown. The most common theories include components of vascular disturbance involving immune complex deposition and microangiopathic changes that lead to collagen degeneration. ${ }^{8}$ Other proposed ideas include collagen production abnormalities and problems with neutrophil migration. ${ }^{2,3,5}$

Generally, in the absence of ulceration or symptoms, it is reasonable not to treat NL given that up to $17 \%$ of lesions may resolve spontaneously but with sequelae. ${ }^{2}$
When ulcerations are present, proper wound care principles are important. Proper wound dressing is needed. When superficial infection is present, systemic antimicrobial therapy or dressings containing antiseptics can be applied. Different advanced dressings have been used successfully in case reports, including bovine collagen, protease modulating products, and ovine forestomach. ${ }^{9-11}$ These products target nonspecific local wound factors such as recruitment of neutrophils, support angiogenesis and reepithelization, and inhibit harmful metalloproteinases. In general, corticosteroids are used as first-line treatment. ${ }^{4,5}$ Topical steroids can decrease inflammation of active lesions. Intralesional and systemic steroids can rapidly halt progression and produce resolution of lesions in most patients. In addition, many studies have treated NL with ultraviolet light therapy and immunomodulators.

Most NL may resolve spontaneously or react to corticosteroid therapy. Unlike these good prognostic wounds, some refractory ulcerative NP can resist therapy without improvement in spite of enough treat time. With extended duration of treatment, patient's quality of life is decreased while medical expenses are increased. Therefore, a new method that not only can decrease therapeutic expenses, but also can achieve better effect, is needed.

Lately, lots of studies pay attention to the effectiveness and the low-risk characteristics of dual-frequency ultrasound application for treating chronic wounds. According to study with Kruglikov and Kruglikova in 2011, it reported 10 cases of patients with chronic venous ulcer treated with dual-frequency ultrasound therapy (LDM) for 8-25 times. ${ }^{7}$ Depending on their study, new concepts of pathophysiology consisting in over-expression of matrix metallo-proteinases (MMPs) in the wound bed and under-expression of heat shock proteins (HSPs) in the wound were proposed. They reported that dual-frequency ultrasound (LDM) with frequencies of 3 and $10 \mathrm{MHz}$ based on simultaneous modulation of MMPs and HSPs in the wound seemed to be an effective alternative treatment for chronic wounds. All patients showed significant clinical outcomes. Chronic wounds became fully epithelized and pain level decreased at least 3 grades according to NRS scale.

The frequency of $L D M^{\circledR}-M E D$ is not constant. It oscilliates between two frequencies such as 3 and $10 \mathrm{MHz}$. A very rapid change of pressure gradients is thought to produce simultaneous modulation of MMPs and HSPs in the wound. ${ }^{7,12}$ Changing frequencies every $5 \mathrm{~ms}$ (200 times per second) is effective in decreasing MMPs and stimulating HSPs, thus helping the wound healing progress. In our case, we found intensive wound granulation after 
the second session of treatment. The patient's wound totally healed and epithelized at the end of the treatment. Because the treatment was pain free without side effects, the patient's satisfaction of the treatment was excellent.

To the best of our knowledge, no study has used ultrasound to treat NL. Our results indicate that the application of $L D M^{\circledR}$-MED seems to be an effective treatment option for NL. Although our results are based on only one case, we expect that more patients with NL could be treated successfully with our LDM ${ }^{\circledR}$-MED protocol. Further large-scale and systematic studies with dual-frequency ultrasound (LDM ${ }^{\circledR}$-MED) therapy are needed to provide innovative formula of treatment for not only NL, but also many kinds of chronic wounds.

\section{ACKNOWLEDGEMENT}

This work was supported by the Soonchunhyang University Research Fund.

\section{CONFLICT OF INTEREST}

The authors report no conflicts of interest.

\section{REFERENCES}

1. Reid SD, Ladizinski B, Lee K, Baibergenova A, Alavi A. Update on necrobiosis lipoidica: a review of etiology, diagnosis, and treatment options. J Am Acad Dermatol 2013;69:783-91.

2. Muller SA, Winkelmann RK. Necrobiosis lipoidica diabeticorum. A clinical and pathological investigation of 171 cases. Arch
Dermatol 1966;93:272-81.

3. Peyrí J, Moreno A, Marcoval J. Necrobiosis lipoidica. Semin Cutan Med Surg 2007;26:87-9.

4. Hocqueloux L, Gautier JF, Lebbe C, Jellal M, Vexiau P, Morel P, et al. [Ulcerated necrobiosis lipoidica associated with insulindependent diabetes mellitus. Beneficial effect of corticosteroid therapy by oral administration]. Presse Med 1996;25:25-7. French.

5. Petzelbauer P, Wolff K, Tappeiner G. Necrobiosis lipoidica: treatment with systemic corticosteroids. Br J Dermatol 1992;126:542-5.

6. Tan E, Patel V, Berth-Jones J. Systemic corticosteroids for the outpatient treatment of necrobiosis lipoidica in a diabetic patient. J Dermatolog Treat 2007;18:246-8.

7. Kruglikov I, Kruglikova E. Dual treatment strategy by venous ulcers: pilot study to dual-Frequency ultrasound application. J Cosmet Dermatol Sci Appl 2011;1:157-63.

8. Sibbald C, Reid S, Alavi A. Necrobiosis lipoidica. Dermatol Clin 2015;33:343-60.

9. Omugha $\mathrm{N}$, Jones AM. The management of hard-toheal necrobiosis with PROMOGRAN. Br J Nurs 2003;12(15 Suppl):S14-20.

10. Simcock JW, Than M, Ward BR, May BC. Treatment of ulcerated necrobiosis lipoidica with ovine forestomach matrix. J Wound Care 2013;22:383-4.

11. Spenceri EA, Nahass GT. Topically applied bovine collagen in the treatment of ulcerative necrobiosis lipoidica diabeticorum. Arch Dermatol 1997;133:817-8.

12. McMurtry AL, Cho K, Young LJ, Nelson CF, Greenhalgh DG. Expression of HSP70 in healing wounds of diabetic and nondiabetic mice. J Surg Res 1999;86:36-41. 